Purpose Given the heterogeneity of clinical presentations, the diagnosis of Systemic Lupus Erythematosus (SLE) can be challenging, in particular in those patients presenting with early or incomplete disease, or with overlapping or atypical features. Autoantibodies (AABs) are important in aiding the clinical diagnosis of SLE, with some few AABs, anti-double-stranded DNA (dsDNA), anti-Smith (Sm), and anti-ribosomal P (riboP) being highly associated with SLE. As none of the traditional AABs has sufficient sensitivity to achieve diagnosis of SLE, current testing is based on measuring multiple $\mathrm{AAB}$ assays either in parallel or serial. We have recently identified novel $\mathrm{AABs}$ in SLE, which hold promise for improving diagnostic testing of SLE (1). We have developed quantitative ELISA-prototypes for five new AABs, which were tested in combination with traditional AABs. The objectives of this study were to evaluate the diagnostic value of novel AABs and to screen for an optimised combination of novel and traditional AABs using logistic regression to increase the diagnostic accuracy of SLE testing.

Methods Serum samples were obtained from 156 SLE patients with European ancestry at the rheumatology department of the Heinrich-Heine University (Düsseldorf, Germany), and Hannover Medical School (Hannover, Germany). SLE samples were compared against 126 samples from autoimmune diseases (AID; myositis: $n=20$; Sjögren's syndrome $(\mathrm{SjS}): \mathrm{n}=31$; rheumatoid arthritis (RA) $n=36$; systemic sclerosis (SSc): $n=39$ ), and 77 healthy control samples. Prototype bead based ELISAs were developed for 5 recently identified novel antigens. Traditional diagnostic AABs were measured using IVD ELISAs and included: SSA/Ro60, SSA/ Ro52, La/SSB, Sm, RNP, dsDNA, Scl70, CENPB, Jo-1, CCP, phospholipid and dsDNA. Optimised marker combinations of new and traditional markers were tested using logistic regression and receiver operating curve analysis (ROC).

Results When comparing 156 SLE patients with 203 control samples, the area under the curve (AUC) of the five novel SLE ELISAs ranged from 0.63 to 0.75 . A cut-off was set at a specificity of $95 \%$ and yielded a sensitivity ranging from $13.5 \%$ to $21.2 \%$ for the five novel assays. The sensitivity and specificity of new ELISAs was comparable to traditional ELISAs, which was in this cohort for anti-dsDNA 35\% and 97\%, anti-Sm 15\% and 97\%, and antiRiboP 26\% and 97\%. A logistic regression model was used to combine the results of multiple tests. Compared to a logistic regression with traditional assays, a logistic regression with novel markers achieved higher sensitivity by pertaining high specificity. The logistic regression model based on a multimarker IVD assay with ten extracted nuclear antigens (ENA) yielded an AUC of 0.87 and a sensitivity of $58 \%$ at a specificity of $95 \%$. By contrast, the optimal combination of traditional and novel ELISAs reached an AUC of 0.92 and a sensitivity of $75 \%$ at a specificity of $95 \%$.

Conclusions This study demonstrates the feasibility of combining test results of novel and traditional AABs using logistic regression to increase the diagnostic accuracy for SLE. Further studies are required to assess the impact of different ethnicities on marker selection and algorithm performance.

\section{PS1:3 ANALYSIS OF C9ORF72 EXPANSIONS IN PATIENTS WITH SYSTEMIC LUPUS ERYTHEMATOSUS AND RHEUMATOID ARTHRITIS: PRELIMINARY DATA}

${ }^{1} \mathrm{M}$ Fredi, ${ }^{2} \mathrm{G}$ Biasiotto, ${ }^{1} \mathrm{I}$ Cavazzana, ${ }^{3} \mathrm{M}$ Filosto, ${ }^{3} \mathrm{~A}$ Padovani, ${ }^{1} \mathrm{~F}$ Franceschini, ${ }^{2}$ Z Zanella. ${ }^{1}$ U.O. Rheumatology and Clinical Immunology, ASST Spedali Civili Brescia, Italy; ${ }^{2}$ Department of Molecular and Translational Medicine and Department of Diagnostic,Spedali Civili and University of Brescia, Italy; ${ }^{3}$ Department of Clinical and Experimental Sciences and Neurology Unit, Spedali Civili and University of Brescia, Italy

\subsection{6/lupus-2018-abstract.52}

Background The most frequent genetic cause of Amyotrophic Lateral Sclerosis (ALS) and Frontotemporal Lobar Dementia (FTLD) is a large hexanucleotide expansion (mostly hundred/ thousand repeats) within a non-coding region of the C9orf72 gene. ${ }^{1}$ The cut-off to distinguish normal and pathogenic expansions has not yet been defined, but most healthy individuals have 2-20 repeats. The pathogenic mechanism of the dominant mutation is most probably toxic gain of functions. Nonetheless, C9orf72 reduced expression has been observed in post-mortem brains of mutated patients. ${ }^{2}$ Interestingly, while gene haploinsufficiency alone seems insufficient to cause neurodegeneration, decreased transcriptional activity with increasing numbers $(>7)$ has been demonstrated in vitro ${ }^{3}$ and knockout mice developped features of systemic lupus erythematosus (SLE). ${ }^{4}$ We investigated C9orf72 gene in a cohort of patients with rheumatoid arthritis (RA) and SLE; as a control group we studied 49 ALS patients without pathogenic expansion.

Methods 29 SLE and 50 RA pts were screened, by the use of a PCR-based protocol, validated in our laboratory. ${ }^{5}$ A cut-off of $\geq 9$ repeat units was considered in our analysis.

Results No patients with large expansions were found. The average and median values of repeat units were 5.29 and 6 in SLE, 4.73 and 2 in RA and 4.8 and 5 in the control population. We individuated $\geq 9$ repeat units in $5 / 30$ (16.7\%) SLE patients and $7 / 50(14 \%)$ RA patients; a prevalence higher than ALS group (8.16\%). We searched for clinical or serological differences among SLE pts with the normal and $\geq 9$ repeat. Although those differences were not statistically significant, we reported a higher prevalence of kidney involvement in patients with a number of repeats $\geq 9(5 / 6 ; 83.3 \%$ vs $7 / 23 ; 30.4 \%)$, $\mathrm{p}=0.056$.

Conclusion Our preliminary results indicate that $\geq 9$ repeats within the C9orf72 gene are detectable in a non negligible number of patients with systemic autoimmune disease, confirming the possible role of C9orf72 in autoimmune system. The possible association with specific subset of disease must be confirmed in a larger cohort of patients.

\section{REFERENCES}

1. Neuron $2011,72: 245-56$.

2. Lancet Neurol 2015,14:291-301.

3. Mol Psychiatry 2016,21:1112-24.

4. Atanasio A, et al. Sci Rep 2016:6:23204.

5. Mol Cell Probes 2017;32:60-64. 